\title{
Wenn der Bagger rollt: Warum politische Konflikte um große Infrastrukturprojekte gewaltsam eskalieren
}

\author{
Sebastian Heidrich $(\mathbb{D} \cdot$ Nils C. Bandelow $\mathbb{1}$
}

Online publiziert: 19. September 2019

(C) Der/die Autor(en) 2019

Zusammenfassung Der Beitrag identifiziert Bedingungen gewaltsamer Eskalation bei Konflikten um Infrastrukturprojekte. Dazu greift er verschiedene Erklärungen aus der Protest- und Bewegungsforschung auf. Empirische Evidenz liefert ein makro-qualitativer Vergleich von 27 Konfliktsituationen bei Infrastrukturprojekten in Deutschland mit der Fuzzy-Set Qualitative Comparative Analysis. Gewaltsame Eskalation setzt die Kombination von Eskalationspotenzial und Eskalationsfunken voraus. Eskalationspotenzial besteht bei grundlegenden Technikkonflikten oder bei der Beteiligung ideologisierter Gruppen. Als Eskalationsfunken wirken Polizeigewalt und der Baustart. Neben dem Verzicht auf Polizeigewalt sollten Eskalationspotenziale und das Timing des Baustarts bei der Eskalationsvermeidung etwa durch Partizipationsprozesse berücksichtigt werden.

Schlüsselwörter Gewalt · Konflikteskalation · Infrastrukturpolitik · Technikkonflikt · Deutsche Politik

Zusatzmaterial online Zusätzliche Informationen sind im Online-Anhang dieses Artikels (https:// doi.org/10.1007/s11615-019-00175-9) enthalten. 


\title{
When the Bulldozers Trundle: Why Infrastructure Policy Conflicts Escalate into Violence
}

\begin{abstract}
This paper identifies conditions of violent escalation related to conflicts about large infrastructure projects. It combines different explanations of protest and social movement research. A macro-qualitative comparative analysis with fuzzy-set qualitative comparative analysis of 27 conflict constellations of large infrastructure projects in Germany provides empirical evidence. Violent escalation results from a combination of the potential for escalation and the existence of a trigger. Fundamental technology conflicts or ideologized groups represent potentials for escalation. Triggers are police violence and the construction start. Beside of avoiding police violence, the potential for escalation and the timing of the construction start should be taken into account by organizing political processes for minimizing escalation.
\end{abstract}

Keywords Violence $\cdot$ Conflict escalation · Infrastructure policy · Technology conflict · German politics

\section{Bedingungen nichtstaatlicher Gewalt als sozialwissenschaftliches Problem}

In der Bundesrepublik Deutschland gibt es mindestens seit den 1970er-Jahren regelmäßig Proteste gegen Großprojekte, ursprünglich auch im Rahmen der AntiAKW-Bewegung. Ein Teil der Proteste verlief friedlich, andere Proteste eskalierten gewaltsam (Neidhardt und Rucht 2001). Diese empirischen Erfahrungen mit eskalierten und nicht eskalierten Konflikten bieten die Möglichkeit zur Identifikation von Eskalationsbedingungen, die Grundlage für eine Bewertung möglicher Deeskalationsstrategien sein kann. Nichtstaatliche Gewalt trat unter anderem bei Protesten gegen große Infrastrukturprojekte auf. Mit dem Konflikt um Stuttgart 21 und den aktuellen Geschehnissen um den Hambacher Forst sind gewaltsame Infrastrukturkonflikte wieder ins Zentrum sozialwissenschaftlichen Interesses und der medialen Aufmerksamkeit gerückt (Brettschneider und Schuster 2013; Kaufer und Lein 2018; Thaa 2013).

Obwohl sich die Grundmuster von Protesten in Deutschland langfristig wenig verändert haben, nehmen gewaltsame Proteste auf insgesamt noch niedrigem Gesamtniveau zu (Rucht und Teune 2017). Diese Studie dient der ersten Identifikation von möglichen Konfigurationen zur Erklärung des Auftretens von Gewalt in diesen Konflikten. Auf Basis des aktuell verfügbaren Datenmaterials werden verschiedene Situationen im Konfliktverlauf mithilfe von Fuzzy-Set Qualitative Comparative Analysis (fsQCA) untersucht.

Zur Einordnung und für das Verständnis gewaltsamer Konflikteskalationen kann der historische Hintergrund früherer Infrastrukturprojekte beitragen. Empirischer Gegenstand sind daher politische Konflikte um große Infrastrukturprojekte in Deutschland zwischen 1970 und 2000.

Theorien der Protest- und Bewegungsforschung fokussieren unterschiedliche Erklärungen von Gewaltphänomenen (Abschn. 2). Die auf Mesoebene angesiedelten 
Erklärungen lassen sich auf eine Mikrofundierung zurückführen (Kausalmechanismus, Abschn. 3). Darauf aufbauend führt der Beitrag die verschiedenen Erklärungen zu einem Modell der gewaltsamen Eskalation von Protest zusammen und leitet Hypothesen ab (Abschn. 4). Der Darstellung der Analyse und Ergebnisse (Abschn. 6) geht eine Diskussion unseres methodischen Ansatzes voran (Abschn. 5). Zuletzt zeigen wir notwendigen Forschungsbedarf auf und kommen zum Fazit, dass die Einbeziehung relevanter Gruppen in Verfahren der frühzeitigen Öffentlichkeitsbeteiligung und konsequent umgesetzte polizeiliche Deeskalationsstrategien Gewalt vermeiden können (Abschn. 7).

\section{Forschungsstand der Protest- und Bewegungsforschung}

Gesellschaftlicher Protest gegen politische Entscheidungen steht im Fokus der Bewegungsforschung. Ihr Hauptaugenmerk liegt dabei auf der Erklärung der Entstehung und Wirkung von Protest. Verschiedene Studien zeigen, dass die Untersuchung und Erklärung von gewaltsamen Eskalationen in politischen Konflikten hingegen eine marginale Betrachtung erfährt (Della Porta 2008; Rucht 2002; Virgl 2011).

Protest kann auf vielfältige Weise geäußert werden. Diese Repertoires of Action (Tilly 1978) adaptieren Erfahrungen aus der Vergangenheit und/oder aus anderen Ländern. In politischen Konflikten um große Infrastrukturprojekte in Deutschland konnte der Protest auf die Erfahrungen mit friedlichen und gewaltsamen Protestformen während der Studentenproteste in den 1960er-Jahren zurückgreifen. Die Wahl für die eine oder andere Protestform basiert dabei nicht allein auf den Entscheidungen der den Protest tragenden Gruppen. Vielmehr ergeben sich die Strategien und Taktiken des Protests in der Interaktion mit der Gegenseite. Dabei erfahren sie eine stetige Anpassung, je nachdem wie sich die Gegenseite verhält und wie erfolgreich die bisherigen Aktionen sind, wie eine Vielzahl empirischer Studien zeigt (Della Porta 1995; McAdam 1983; Waldmann 1993; White 1993).

Eine genauere Betrachtung der politischen Strukturen und Prozesse ermöglicht der Political-Opportunity-Structure-Ansatz (Kriesi 1989, S. 295). Er zeigt, dass geschlossene und instabile politische Systeme gewaltsame Eskalation begünstigen (Bosi 2006; Koopmans et al. 2008). Innerhalb eines politischen Systems beeinflussen unterschiedliche Bedingungen den Protest. Dazu zählen vom Protest kaum beeinflussbare Ereignisse (Goodwin und Jasper 1999, S. 40). In Konflikten um große Infrastrukturprojekte bieten die im Prozess der Genehmigung implementierten Beteiligungsinstrumente (bspw. in den Raumordnungs- oder Planfeststellungsverfahren) Möglichkeiten der friedlichen Einflussnahme. Im Verlauf von Konflikten entfallen diese formellen Einflussmöglichkeiten. Gewalt kann dann als legitime Protestform erscheinen. Eine weitere Möglichkeit für die Wahl von Gewalt bieten dynamische Ereignisse. Dann fällt dem Protest-Policing und eskalativen Polizeistrategien wie dem Einsatz von Polizeigewalt eine besondere Bedeutung zu (Della Porta und Reiter 1998). Unklar bleibt, welche Bedingungen in welchen Konstellationen entscheidend für die Wahl für oder gegen Gewalt sind.

Mit dem Ressource-Mobilization-Ansatz der Protestforschung treten Protestgruppen und die von ihnen direkt beeinflussbaren Bedingungen in das Zentrum. Die Wahl 
für eine bestimmte Protestform bedarf nicht nur bestimmter Political-OpportunityStructures, sondern auch zur Realisierung notwendige Ressourcen. In dieser Perspektive entsteht Gewalt, wenn Gruppen die notwendigen Ressourcen für friedlichen Protest nicht aufbringen, aber auf Gewaltressourcen zurückgreifen können (Tilly 1978; Zimmermann 1998). Gewalt ist dabei eine Alternative zur friedlichen Durchsetzung von Interessen. Hinzu kommt, dass die verschiedenen Gruppen hinsichtlich der verfügbaren Ressourcen in Konkurrenz zueinander stehen. Verschiedene Studien zeigen, dass sich Gewaltgruppen innerhalb von sozialen Bewegungen bilden können und sich von diesen im Zeitverlauf lösen (Irvin 1999; Zwerman et al. 2000).

Gewalt muss nicht nur gegenüber anderen, sondern auch im Selbstverständnis gerechtfertigt werden (Beier 2016; Lamnek 2002). Als Legitimationsinstrument können die von Identitäts- und Framing-Ansätzen der Bewegungsforschung fokussierten Frames und den Gesamtkontext beschreibende Narrative dienen, welche Wissen darüber bereitstellen, wie die Welt funktioniert und welche Konsequenzen sich daraus ergeben (Snow et al. 1986; Snow und Oliver 1995; Taylor und Whittier 1992). Grundlage für die Entwicklung von gewaltfördernden Frames und Narrativen sind die Interaktionen im Konfliktverlauf. Hinzu kommen Weltanschauungen, die von den Protestgruppen und ihren Mitgliedern vertreten werden und die Legitimität politischer Entscheidungsprozesse hinterfragen. Durch selbstreferentielle Gruppenprozesse kann dies soweit gestärkt werden, dass sich im Laufe von Eskalationsprozessen der Legitimationsglaube gewaltsamer Handlungen entwickelt (Arendt 1995; Della Porta 2013; Enzmann 2013).

Alle Perspektiven und bisherigen empirischen Befunde verbindet, dass sie nur einzelne Konflikte oder ähnlich gelagerte Fälle und deren gewaltsame Eskalation erklären können. Gleichzeitig werden die Mikrofundierungen der Ansätze selten expliziert, sodass eine Verbindung der Ansätze erschwert wird.

Mit dem Emotional Turn in der Politikwissenschaft hielten Emotionen auch Einzug in die Forschung zu Protestbewegungen (Goodwin et al. 2001; Jasper 1998, 2006). In der Erforschung gewaltsamer Eskalationen finden sich diese jedoch kaum wieder. Zwar sprechen Vertreterinnen und Vertreter des Framing-Ansatzes von der emotionalen Kraft, die Frames und Narrativen innewohnt, explizite Auswirkungen auf die zugrunde liegende Handlungstheorie oder auf das Forschungsprogramm hat dies allerdings nicht. Dabei ermöglicht die Theorie der begrenzten Rationalität die Implementierung von Emotionen explizit. In der Forschungstradition der Frustrations-Aggressions-Theorie spielen Affekte eine zentrale Rolle. Das kognitiv-neoassoziationistische Aggressionsmodell (Berkowitz 1993) ist dabei ein Ausgangspunkt für die Mikrofundierung der Zusammenhänge auf der Mesoebene und ermöglicht die Verbindung der Ergebnisse. Aus diesem Grund stellen wir in den folgenden Abschnitten diese Perspektive näher vor und entwickeln auf dessen Basis ein theoretisches Modell zur Erklärung gewaltsamer Eskalationen von Protest. 


\section{Mikrofundierung gewaltsamer Eskalation: Das kognitiv- neoassoziationistische Aggressionsmodell}

Kollektive Gewaltanwendungen im Rahmen von Protesten gegen große Infrastrukturprojekte lassen sich am besten verstehen, wenn die Erklärung theoretisch auf die Individualebene zurückgeführt wird (Coleman 1994). Diese Mikrofundierung dient der Verbindung der zu untersuchenden Phänomene auf der Mesoebene und der Begründung, wie die Startbedingungen eines Bauprozesses gewaltsamen Protest verursachen. Sie ist damit der kausale Mechanismus, der Antezedens und Explanandum miteinander verbindet. Notwendig ist dieses Konstrukt, da Phänomene auf der Mesoebene nicht direkt, sondern immer über die Individualebene miteinander verbunden sind. Wir untersuchen somit empirisch auf der Mesoebene, welche Startbedingungen vorliegen müssen, damit sich das Phänomen Protestgewalt realisiert. Die Individualebene ist dagegen nicht Gegenstand unserer Untersuchung und wird keinem Test unterzogen. Sie dient vielmehr dem Verständnis gewaltsamer Eskalation und wird als kausaler Mechanismus angenommen.

Das kognitiv-neoassoziationistische Aggressionsmodell als Teil der sozialpsychologischen Forschung zu Gewaltphänomenen postuliert unangenehm und aversiv wahrgenommene Ereignisse als Ausgangspunkt gewaltsamer Handlungen auf der Individualebene (Berkowitz 1993, S. 48-56). Diese bewirken einen unspezifischen negativen Affekt, welcher automatisch verschiedene motorische Reaktionen, Gefühle, Gedanken und Erinnerungen aktiviert und in der Konsequenz u. a. mit Aggression verbundene Reaktionen nach sich zieht. Hierzu zählen die Fokussierung auf ein verfügbares Ziel und die Absicht, jemanden zu verletzen oder ein Objekt zu beschädigen. Bewusste und tiefergehende, komplexe kognitive Prozesse finden demnach erst im Anschluss daran statt.

Das Individuum attribuiert das Ereignis auf Basis seiner Erfahrungen, seines Wissens und seiner Einstellung. Es werden Szenarien über mögliche Auswirkungen von Handlungen entworfen. Gruppenprozesse spielen somit vor und nach einem unangenehmen und aversiv erlebten Ereignis eine zentrale Rolle, da sie diese kognitiven Prozesse insbesondere in politischen Konflikten maßgeblich beeinflussen. Ungerechtigkeitsnarrative und -frames können durch Wiederholung im Konfliktverlauf internalisiert werden, sodass sie diesen automatisch ablaufenden Prozessen zugeordnet und abgerufen werden. Im Nachgang finden Attribuierung und Interpretation dieser Ereignisse ebenso durch selbstreferentielle Gruppenprozesse statt. Wie stark der Einfluss dieser verschiedenen Mechanismen auf die tatsächlich realisierte Reaktion ist, hängt von der zur Verfügung stehenden Zeit ab und davon, wie ,nah“ ein aversives Ereignis aus Sicht des Individuums ist (impulsive Reaktion versus strategisches Handeln).

Beeinflussen die genannten Bedingungen die individuelle Absicht gewaltvoll zu handeln, wirken zwei weitere Bedingungen auf die tatsächliche Realisierung dieses Vorhabens. Zum einen mindert die Aussicht auf Bestrafung durch die Gesellschaft (Strafverfolgung) aggressives Verhalten. Zum anderen ist aggressives Verhalten von Bezugsgruppen des Individuums abhängig. Insgesamt können diese Bedingungen als Kosten-Nutzen-Überlegungen interpretiert werden (Schmid 2002). 


\section{Modell und Hypothesen der gewaltsamen Eskalation von Protest}

Für die empirische Analyse verbinden wir einzelne Ergebnisse der Forschung auf Basis der Mikrofundierung zu einem theoretischen Modell der gewaltsamen Eskalation von Protest in Konflikten um große Infrastrukturprojekte, ehe wir im folgenden Kapitel näher auf die verwendete Methodik eingehen. Das Modell zielt auf Erklärungen auf der Mesoebene. Diese können oft, aber nicht immer zwingend auf Mikroerklärungen zurückgeführt werden (Mayntz 2009, S. 97-122). In unserem Modell wird die Mikroebene angelehnt an Coleman (1994) als Unterbau aufgenommen, um die wirksamen Mechanismen zu illustrieren. Grundlage dieser Verbindung sind die Annahmen des Konzeptes der begrenzten Rationalität. Auf dieser Basis lässt sich das in Abb. 1 dargestellte Modell entwickeln.

Der Ausgangspunkt für die gewaltsame Eskalation von Protest sind Political Opportunity Structures, die Gewalthandlungen für den Protest zur Zieldurchsetzung attraktiv erscheinen lassen, da sie direkt das Ziel der Verhinderung des Infrastrukturprojektes bedrohen (Goodwin und Jasper 1999, S. 40). Im Prozess der Realisierung von Infrastrukturprojekten lassen sich Momente finden, die diesem Kriterium genügen (bspw. Vorstellung der Projektidee, Baustart). Sie wirken als Symbol für den Fortschritt des Projektes. Eine ebenso symbolische Wirkung in Bezug auf den Protest hat der Einsatz von Gewalt durch die Polizei. Für die Protestbewegung bieten diese, in der Erwartung der Zielerreichung oder der Vermeidung des Scheiterns, Anreize zu kollektivem Handeln.

Betrachtet werden damit Ereignisse, bei denen friedliche Möglichkeiten ausgeschöpft scheinen und daher Gewalt als Mittel der Interessendurchsetzung opportun sind. Daneben ist auf Basis des Ressource-Mobilization-Ansatzes eine weitere Voraussetzung notwendig: Gewalthandlungen brauchen verschiedene Ressourcen. Dazu zählen unter anderem gewaltlegitimierende Ressourcen in Form von Werten

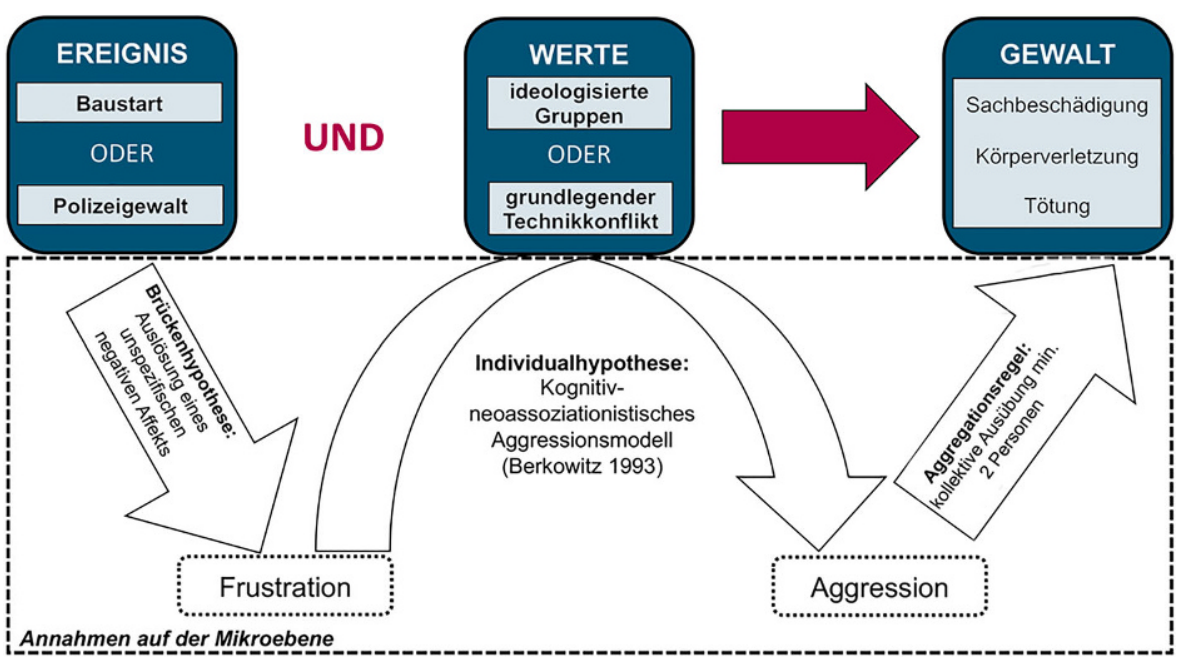

Abb. 1 Modell der gewaltsamen Eskalation von Protest. (Quelle: Eigene Darstellung) 
auf Seiten des Protestes, die im Kontrast zu denen der Projektbefürworter stehen. Dieser Gegensatz der Werte- und Normensysteme bedingt die Dämonisierung der Gegenseite und klare Freund-Feind-Zuordnungen (Glasl 2013; Bandelow und Thies 2014; Vogeler und Bandelow 2016, 2018) sowie die Infragestellung der Legitimität von Entscheidungsprozessen. Entsprechend lässt sich Protestgewalt zum Zeitpunkt der oben beschriebenen Ereignisse legitimieren. Wertegegensätze sind durch unterschiedliche Phänomene messbar. Erstens repräsentieren grundlegende Technikkonflikte das Aufeinandertreffen stark gegensätzlicher Werte und Normen. Ein Beispiel ist die Atomenergie. Zweitens repräsentieren ideologisierte Gruppen in Konflikten um große Infrastrukturprojekte solche Werte- und Normensysteme. Die Beteiligung dieser Gruppen an Protesten gegen große Infrastrukturprojekte, ihr Werte- und Normensystem und Radikalisierungstendenzen im Konfliktverlauf (Della Porta 2013; Glasl 2013) begünstigen die Reduktion von Sanktionsdruck innerhalb der Gruppe und befördern den Wechsel von friedlichen zu gewaltsamen Protestformen.

Gewaltsamer Protest wird damit immer durch die Kombination von Bedingungen aus zwei Gruppen verursacht. Zum einen ist ein Ereignis notwendig, welches die Anwendung von Gewalt für den Protest zielführend erscheinen lässt. Ein einzelnes Ereignis wie Baustart stellt allerdings keine notwendige Bedingung da, weil auch andere Ereignisse wie die Anwendung von Polizeigewalt Element einer hinreichenden Kombination sein kann. Zum anderen ist eine Ressource notwendig, um Protestgewalt gegenüber den verschiedenen Protestgruppen und der Öffentlichkeit zu legitimieren. Auch hier gilt, dass einzelne Bedingungen aus der Gruppe der gewaltlegitimierenden Ressourcen nicht notwendig gewaltsame Eskalation des Protestes verursachen. Nur in ihrer jeweiligen Gesamtheit sind sie eine notwendige Voraussetzung für die gewaltsame Eskalation von Protesten. Die Bedingungen innerhalb der Gruppen fungieren damit als funktionale Äquivalente. Entsprechend lassen sich für die Analyse äquifinale Pfade formulieren, die Protestgewalt jeweils hinreichend erklären und es in der weiteren Untersuchung zu testen gilt:

Hypothese 1 Die Beteiligung von ideologisierten Gruppen (I) zum Zeitpunkt des Baustarts (B) verursacht gewaltsamen Protest (G). Formalisiert: I * B $\rightarrow$ G.

Hypothese 2 Bei Vorliegen eines grundlegenden Technikkonflikts (T) verursacht der Baustart (B) die gewaltsame Eskalation des Protestes (G). Formalisiert: $\mathrm{T} *$ $\mathrm{B} \rightarrow \mathrm{G}$.

Hypothese 3 Polizeigewalt $(\mathrm{P})$ verursacht bei Teilnahme von ideologisierten Gruppen (I) am Konflikt gewaltsamen Protest (G). Formalisiert: $\mathrm{P} * \mathrm{I} \rightarrow \mathrm{G}$.

Hypothese 4 In einem Technikkonflikt $(T)$ verursacht der Einsatz von Polizeigewalt (P) die gewaltsame Eskalation des Protestes (G). Formalisiert: $\mathrm{T} * \mathrm{P} \rightarrow \mathrm{G}$.

Für die vollständige Beschreibung der Kausalität von gewaltsamen Protesten in Konflikten um große Infrastrukturprojekte in Deutschland ist neben der Nennung und empirischen Messung der Startbedingungen und Wirkung, der auf der Mikroebene wirksame Kausalmechanismus zu beschreiben (Tilly 2001). Das oben dargestellte kognitiv-neoassoziationistische Aggressionsmodell und die zugehörige Frustrations- 
Aggressions-Theorie kann als allgemeine Gesetzmäßigkeit auf die hier zu untersuchenden Infrastrukturkonflikte angewandt werden, da diese Spezialfälle darstellen. Die Individualebene lässt sich mit der Mesoebene verbinden, da zum einen angenommen werden kann, dass die verschiedenen relevanten Dispositionen im Aggregat normalverteilt sind (Hirschle 2015, S. 23-24) und daneben bereits zwei Personen ausreichen, um den Protest als gewaltsam zu kategorisieren. Dies deckt sich mit der medialen Berichterstattung. Zum anderen helfen Transformations- und Aggregationsregel dabei, die verschiedenen Ebenen miteinander zu verbinden (vgl. Abb. 1).

\section{Methodik}

Der Analyse liegen mehrere Annahmen zugrunde, welche die Wahl der hier verwendeten Methode bedingt: Fälle werden als Konfigurationen unterschiedlicher Bedingungen verstanden. Die Wirkung (Outcome) kann durch verschiedene Konfigurationen äquifinal ausgelöst werden. Die hierfür relevanten Bedingungen sind dabei notwendig oder hinreichend. Gleichzeitig spielt der Kontext eines jeden Falles eine bedeutende Rolle, sodass der Fokus auf den Fällen liegt. Um die tatsächlichen Ursachenstrukturen im Hinblick auf die zu untersuchende Forschungsfrage zu identifizieren, muss eine Methodik angewendet werden, die durch Fallvergleich einzelne Bedingungen ,eliminiert“. Auf Basis dieser Annahmen hat Charles C. Ragin (1987) die Methode des makro-qualitativen Vergleichs (QCA) entwickelt, die in dieser Untersuchung zur Anwendung kommt.

\subsection{Crisp-Set, Temporal/Time-Series oder Fuzzy-Set-QCA?}

In Form von Crisp-Set-QCA (csQCA) wird analysiert, ob das Vorliegen einer Bedingung den Outcome bewirkt. Diese ursprüngliche Version dichotomisiert Bedingungen und minimiert Konfigurationen mithilfe boolescher Algebra. Sie bezieht Bedingungen statisch in die Analyse ein ohne die zeitliche Abfolge der Bedingungen zu erheben. Dies hat in der Vergangenheit zu Kritik geführt, da dynamische Prozesse nicht untersucht werden können. Zur Untersuchung solcher Fälle wurde csQCA durch eine veränderte Konstruktion der Bedingungen erweitert. Mit Temporal-QCA (Caren und Panofsky 2005; Ragin und Strand 2008), Time Series QCA (Hino 2009) und Temporal Necessary Condition Analysis (Dul et al. 2010; Hak et al. 2013) bieten sich neue Möglichkeiten, dynamische Prozesse der Realisierung eines Outcomes zu untersuchen und mithilfe von QCA zu analysieren. In der vorliegenden Untersuchung fällt der zeitlichen Dimension aber keine Relevanz zu, da Situationsstrukturen untersucht werden.

Neben dem Aspekt der Zeit wurde die Einschränkung der Analyse von komplexen sozialen Phänomenen durch die Dichotomisierung der Bedingungen in csQCA kritisiert und führte zur Entwicklung von Fuzzy-Set-QCA (Ragin 2000). Grundlage hierfür ist die Verwendung von Fuzzy-Logik und damit ein verändertes Mengenkonzept. Eine relevante Frage für die Wahl der Methode ist die, ob dem Outcome ein Konzept zugrunde liegt, welches die eine oder andere Methode nach sich zieht. 
In unserer Untersuchung verwenden wir fsQCA, da sowohl der Outcome als auch einzelne Bedingungen mithilfe von Fuzzy-Mengen operationalisierbar sind.

\subsection{Operationalisierung und Datenerhebung}

Durch die Verwendung von fsQCA ergibt sich, dass die zu erhebenden Bedingungen so operationalisiert werden müssen, dass die Bedingungen Werte zwischen 0 und 1 annehmen können. 1 zeigt dabei an, dass ein Fall vollständiger Teil einer Bedingung ist. Der Wert 0 bedeutet hingegen, dass der Fall vollständiger Teil des Komplements ist. Fuzzy-Werte unterscheiden sich vom Mengenkonzept der Dichotomie in csQCA insofern, dass Fälle zu einem bestimmten Grad gleichzeitig Element einer Menge und dessen Komplements sind. Der Anker von 0,5 zeigt dabei die Grenze, zu der der Fall eher der einen oder der anderen Menge gehört. Beispielsweise bedeutet ein Fuzzy-Wert von 0,7, dass ein Fall eher die Eigenschaft einer Bedingung aufweist. Gleichzeitig besitzt dieser Fall mit 0,3 wenig die Eigenschaft des Komplements. Die Dichotomie von csQCA sind insofern ein Spezialfall in fsQCA und können mit dieser Methode entsprechend verarbeitet werden.

Das theoretische Modell der gewaltsamen Eskalation von Protest beinhaltet die Konstrukte Ereignis und Werte sowie den Outcome Protestgewalt. Er liegt vor, wenn mindestens zwei Personen kollektiv absichtlich oder leichtfertig Gegenstände beschädigen und/oder Personen verletzen oder töten (Graham und Gurr 1969; Neidhardt 1986) und dem Protest zugeordnet werden können. Die Messung des Outcomes orientiert sich zunächst an den theoretischen Konzepten der PRODATDatenbank (Variable form_r6). Ergänzend nutzt die Messung Konzepte des deutschen Strafrechts, da dieses erstens die normativen Grundlagen des Gemeinwesens widerspiegelt und zweitens auch für mögliche Sanktionen gegenüber den Täterinnen und Tätern relevant ist. Die Skala bildet auf einer Dimension ab, wie sehr ein Verhalten darauf ausgerichtet ist, auch unter Inkaufnahme der Schädigung der Gegenseite, das eigene Ziel zu erreichen. Wir verwenden dabei anlehnend an die Gewaltunterscheidung in PRODAT und entsprechend der guten Praxis von fsQCA eine Vierer-Skala.

1 bedeutet, dass in einem Fall der Protest in Gewalt vollständig eskaliert ist. Dies liegt vor, wenn dem Protest schwere Sachbeschädigung oder Körperverletzung oder Tötung zugeordnet werden kann (form_r6=6, schwere Gewalt). Fälle weisen eher Protestgewalt auf, wenn dem Protest leichte Sachbeschädigung oder Körperverletzung zuzuordnen ist (form_r6 $=5$, leichte Gewalt). Keine Protestgewalt stellen Handlungen dar, die weder Sachen noch Personen direkt schädigen. Sie werden entsprechend mit 0 kodiert (form_r6 $<4$, appellativ, prozedural, demonstrativ). Dies ist bei friedlichen Protestformen wie dem Sammeln von Unterschriften, dem Verteilen von Flugblättern oder friedlichen Demonstrationen der Fall. Friedliche Blockaden werden durch das Einwirken auf andere als wenig gewaltvoll und somit mit 0,3 kodiert (form_r6=4, konfrontativ).

Die Bedingung Polizeigewalt lässt sich nicht analog zum Outcome kodieren, da die Daten nicht gleichermaßen verfügbar sind und (vor allem) die rechtlichen Grundlagen zwischen etwa der Bewertung von Straßensperren durch die Polizei einerseits und durch Protestierende andererseits verschieden sind. Werte unterhalb 
von 0,5 müssen immer vergeben werden, wenn Polizeiaktionen nicht gezielt einen Schaden bei den Protestierenden in Kauf nehmen. Unterhalb dieser Schwelle sind zwar theoretisch Differenzierungen denkbar, sie werden aber nicht systematisch in Medien berichtet und können daher nicht kodiert werden. Alle Fälle „ohne“ entsprechende Polizeigewalt sind daher hier mit 0 kodiert. Der Wert 0,6 wird vergeben, wenn Schäden an Protestierenden in Kauf genommen, aber nicht beabsichtigt werden (etwa bei einer Räumung festgeketteter Demonstranten). Alle Polizeieinsätze, die auf Schädigung von Protestierenden zielen, aber keine Tötung in Kauf nehmen (also Wasserwerfer, Schlagstöcke etc.) werden mit 0,8 kodiert. Dabei sind schwere Körperverletzungen möglich, wie das spätere Beispiel Stuttgart 21 zeigt. Der Wert 1 ist den theoretischen Fällen von Einsätzen mit Inkaufnahme von Tötungen (etwa Verwendung von Schusswaffen) vorbehalten.

Bei der Bedingung ideologisierte Gruppen geht es um die ideologisch begründete grundsätzliche Infragestellung der Legitimität von Entscheidungsprozessen im politischen System. Daraus resultiert ein Konfliktpotenzial, mit dem Protestgewalt legitimiert wird. Der Wert 0 steht daher für Anwohner, bei denen es keinen Hinweis auf grundsätzliche Unterschiede im Wertesystem gibt. Institutionalisierte Umweltschutzverbände werden mit 0,3 kodiert, da sie aufgrund ihrer rechtlichen Struktur zwar auch klare Feindschaften verankert haben können, Gewalt allerdings aufgrund der Logik unseres politischen Systems und des Mediensystems keine Handlungsalternative darstellt und dementsprechend innerhalb dieser Gruppen entsprechende Narrative wirken. Kleine, lose radikalisierte Gruppen wie nicht institutionalisierte Umweltgruppen beim Großflughafen München sprechen den konventionellen Entscheidungsprozessen die Legitimität $a b$, wenn diese mit höherrangigen Gütern wie Nachhaltigkeit kollidieren. Bei diesen Gruppen findet ein Abwägen zwischen dem Grad der Gewalt und dem zu erreichenden Ziel statt. Sie werden daher mit 0,7 kodiert. K-Gruppen, extremistische Parteien und militante Gruppen stehen dagegen in radikaler Gegnerschaft zum politischen System. Den politischen Prozessen wird ihre Legitimität abgesprochen. Sie werden daher mit 1 kodiert.

Die Bedingung Baustart wird hingegen aufgrund seiner Eindeutigkeit dichotomisiert. Mit 1 sind Fälle codiert, sofern in der Wahrnehmung des Protestes der Baustart vorlag. Dafür reichen wie in Gorleben auch Probebohrungen oder wie in München der Bau einer Zufahrtsstraße aus, um als Baustart wahrgenommen zu werden, auch wenn der Bau des konkreten Projektes noch nicht begonnen hat. Die Bedingung grundlegender Technikkonflikt liegt vollständig vor, wenn der Konflikt gesamtgesellschaftlich als solcher wahrgenommen wird. Mit 0,7 werden Fälle kodiert, in denen die gewaltausübende Gruppe selbst die verwendete Technologie prinzipiell ablehnt, während in der Gesellschaft kein Konflikt über die Verwendung der Technologie existiert. Dies ist bei einer Umweltgruppe beim Großflughafen München der Fall.

Für alle Fälle wurden Fallstudien zu den jeweiligen Infrastrukturkonflikten angefertigt, um Fallwissen zu generieren und die Relevanz der zu untersuchenden Bedingungen zu überprüfen. Diese basieren auf Sekundäranalysen von Studien zu den Fällen und systematischen Medienanalysen (Frankfurter Allgemeine Zeitung über F.A.Z.-Research, Hamburger Abendblatt, Ostsee-Zeitung, Süddeutsche Zeitung über 
Onlinerecherche). Zeitlich und lokal konnte die Suche durch die Protestdatenbank PRODAT (Rucht und Teune o.J.) eingeschränkt werden.

\subsection{Fallauswahl}

Die Auswahl der Fälle erfolgt in zwei Schritten und folgt dem Prinzip der größtmöglichen Heterogenität. Dieses Prinzip wird gewählt, da die Mikrofundierung des theoretischen Modells eine Logik der Entstehung von Aggression postuliert, die auf ganz unterschiedliche Fälle gleichermaßen anwendbar ist. Um die Anzahl der zu untersuchenden Bedingungen und Fälle zu begrenzen und eine Fallorientierung zu gewährleisten, beschränken wir uns auf politische Konflikte um große Infrastrukturprojekte in Deutschland zwischen 1970 und 2000. Dies hat mehrere Gründe: Bei politischen Konflikten um große Infrastrukturprojekte sind anfänglich oft materielle Konflikte und NIMBY-Motive (,Not In My Back Yard') für die vor Ort Betroffenen ausschlaggebend. Erst im weiteren Verlauf kann sich der Konflikt auf NIABYMotive (,Not In Anybody's Back Yard') ausweiten und damit überregionale, nicht direkt Betroffene zum Protest motivieren, sodass sich ein Wandel der Konfliktsituation feststellen lässt. Die zeitliche Begrenzung erfolgt, da zum einen Proteste gegen große Infrastrukturprojekte erst mit dem Ende des wirtschaftlichen Aufschwungs und dem Aufkommen der Umweltbewegung in den 1970er-Jahren populär wurden. Zum anderen kann die Relevanz der Effekte von Internet, Social Media und digitalen Verbreitungswegen von Frames durch die Begrenzung auf 2000 in der Analyse neutralisiert werden, da sie bis zu dieser Zeit keine Rolle spielten.

Im ersten Schritt werden über PRODAT anhand von Protestereignissen Infrastrukturkonflikte in Deutschland zwischen 1970 und 2000 identifiziert. Da Widerstand gegen das Projekt eine Grundbedingung zur Anwendung des theoretischen Modells darstellt, werden zuerst nur Konflikte mit mindestens zehn Protestereignissen aufgenommen, um einen Überblick über polarisierte Konflikte zu erhalten. Gleichzeitig werden die Anzahl der in Gewalt eskalierten Protestereignisse ver-

Tab. 1 Untersuchte Infrastrukturprojekte. (Quelle: PRODAT, eigene Darstellung; Fallauswahl: theoriebasiert)

\begin{tabular}{lll}
\hline Infrastrukturprojekt & $\begin{array}{l}\text { Anzahl der Pro- } \\
\text { testereignisse in } \\
\text { PRODAT }\end{array}$ & $\begin{array}{l}\text { Anzahl der in } \\
\text { Gewalt eskalierten } \\
\text { Protestereignisse }\end{array}$ \\
\hline Startbahn West & 93 & 46 \\
$\begin{array}{l}\text { Atominfrastruktur } \\
\text { Gorleben }\end{array}$ & 52 & 11 \\
$\begin{array}{l}\text { Atomkraftwerk Wyhl } \\
\text { Großflughafen Mün- } \\
\text { chen }\end{array}$ & 15 & $0^{\text {a }}$ \\
$\begin{array}{l}\text { Ostsee-Autobahn A20 } \\
\text { Transrapid Hamburg- }\end{array}$ & 9 & 1 \\
Berlin & 9 & 1 \\
Transitautobahn A24 & 8 & 0 \\
Flughafen Stuttgart & 4 & 0 \\
\hline
\end{tabular}

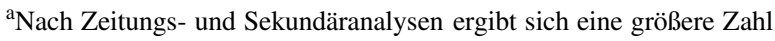
von Gewaltereignissen 
Tab. 2 Fälle ${ }^{\mathrm{a}}$. (Quelle: Eigene Daten)

\begin{tabular}{|c|c|c|c|c|c|c|}
\hline Fall & Zeitraum & $\begin{array}{l}\text { START- } \\
\text { CONS }\end{array}$ & POLVIO & IDEOLOG & $\begin{array}{l}\text { TECH- } \\
\mathrm{CON}\end{array}$ & $\begin{array}{l}\text { VIO- } \\
\text { LENCE }\end{array}$ \\
\hline A20 I & 1991 & 0 & 0 & 0,3 & 0 & 0 \\
\hline A20 II & 1992-30.04.1994 & 0 & 0,6 & 0,3 & 0 & 0,3 \\
\hline A20 III & 01.05.1994-31.05.1994 & 1 & 0 & 1 & 0 & 1 \\
\hline A20 IV & 01.06.1994-1997 & 0 & 0 & 0 & 0 & 0 \\
\hline A24 I & 1978 & 0 & 0 & 0 & 0 & 0 \\
\hline A24 II & 1979-1981 & 0 & 0 & 0 & 0 & 0 \\
\hline A24 III & $01 .-28.02 .1981$ & 1 & 0 & 0 & 0 & 0 \\
\hline A24 IV & 1981-1982 & 0 & 0 & 0 & 0 & 0 \\
\hline Stuttgart I & $30.06 .1976-18.04 .1978$ & 0 & 0 & 0 & 0 & 0,3 \\
\hline Stuttgart II & 19.04.1978-1983 & 0 & 0 & 0,7 & 0 & 0,3 \\
\hline Gorleben I & $22.02 .1977-28.06 .1978$ & 0 & 0 & 1 & 1 & 0 \\
\hline Gorleben II & 29.06.1978-13.03.1979 & 0 & 0 & 1 & 1 & 0 \\
\hline Gorleben III & 14.03.1979-1985 & 1 & 0,8 & 1 & 1 & 1 \\
\hline München I & $1967-31.05 .1979$ & 0 & 0 & 0 & 0 & 0,7 \\
\hline München II & 01.06.1979-22.09.1979 & 0 & 0 & 0,7 & 0 & 0 \\
\hline München III & 23.09.1979-31.12.1979 & 0 & 0 & 0,7 & 0 & 0 \\
\hline München IV & 01.01.1980-31.01.1980 & 1 & 0 & 0,7 & 0,7 & 1 \\
\hline München V & 01.02.1980-1992 & 0 & 0 & 0,7 & 0 & 0 \\
\hline Startbahn West I & $1965-1968$ & 0 & 0 & 0 & 0 & 0 \\
\hline Startbahn West II & $1968-1970$ & 0 & 0 & 0 & 0 & 0 \\
\hline $\begin{array}{l}\text { Startbahn West } \\
\text { III }\end{array}$ & $1970-1980$ & 0 & 0 & 1 & 0 & 0 \\
\hline $\begin{array}{l}\text { Startbahn West } \\
\text { IV }\end{array}$ & 01.01.1980-1982 & 1 & 0,8 & 1 & 0 & 1 \\
\hline Transrapid $\mathrm{HH}$ & $15.07 .1992-05.02 .2000$ & 0 & 0 & 0 & 0 & 0 \\
\hline Wyhl I & 1973-22.01.1975 & 0 & 0 & 0 & 1 & 0 \\
\hline Wyhl II & 22.01.-16.02.1975 & 0 & 0 & 0 & 1 & 0 \\
\hline Wyhl III & 17.02.-19.02.1975 & 1 & 0 & 0 & 1 & 0,7 \\
\hline Wyhl IV & 20.02.-24.02.1975/1983 & 0 & 0,8 & 1 & 1 & 0,7 \\
\hline \multicolumn{2}{|c|}{ Gesamtzahl Fälle $>0,5$} & 6 & 4 & 12 & 8 & 7 \\
\hline
\end{tabular}

STARTCONS = Baustart, POLVIO = Polizeigewalt, IDEOLOG = Beteiligung ideologisierter Gruppen, TECHCON = grundlegender Technikkonflikt, $O U T C O M E=$ kollektive Protestgewalt

${ }^{a}$ Der Rohdatensatz kann wegen des Umfangs nicht dargestellt werden. Auf Anfrage wird dieser gerne zur Verfügung gestellt

zeichnet (Online-Anhang A). Die Auswahl der zu untersuchenden Konflikte ergibt sich aus der Anzahl der Protestereignisse und der Anzahl der in Gewalt eskalierten Protestereignisse. Hinzu kommt, dass die Konflikte die Infrastrukturarten Flughafen, Straße, Atomenergie und Schiene repräsentieren sollen, da diese Technologien auf unterschiedliche Akzeptanz in der Bevölkerung treffen (Tab. 1). Unter Anwendung dieser Kriterien nehmen wir die Konflikte Startbahn West, Atominfrastruktur Gorleben, Atomkraftwerk Wyhl, Großflughafen München, Ostsee-Autobahn A20, Transrapid, Transitautobahn A24 und Flughafen Stuttgart in die Untersuchung auf. Anschließend werden in einem zweiten Schritt, basierend auf den Case Studies 
und der Regel, dass eine veränderte Bedingung eine neue Situation erzeugt, Fälle erhoben, welche neue Konfliktsituationen repräsentieren und in denen der Protest Handlungsentscheidungen trifft.

Es ist allerdings unter Verwendung der Case Studies zu prüfen, inwiefern sich die jeweiligen Konstellationen tatsächlich als voneinander unabhängige Fälle interpretieren lassen. Zusätzlich muss überprüft werden, ob es in den jeweiligen Infrastrukturprojekten Ereignisse gegeben hat, die auf mehrere von uns untersuchten Konstellationen eingewirkt haben. Diese Untersuchung ist vor allem bei den Fällen notwendig, bei denen es wiederholt zu Gewaltanwendungen gekommen ist. Das gilt erstens für den Konflikt um das Zwischenlager in Gorleben. Hier zeigt die qualitative Betrachtung, dass verschiedene Gewalteskalationen miteinander zusammenhängen. Wir haben daher alle Ereignisse mit Gewalteskalation als eine zusammenhängende Phase interpretiert und für diese Phase die Bedingungen zu Beginn der Phase kodiert. Ähnliches gilt für den Konflikt Startbahn West. Auch hier findet sich wiederholte gewaltsame Eskalation, die aber nicht unabhängig voneinander ist, sondern es sinnvoll erscheint, auch hier von einer andauernden Phase auszugehen. Somit ergeben sich $27 \mathrm{zu}$ untersuchende Fälle (Tab. 2).

\section{Konfigurationen für die gewaltsame Eskalation von Konflikten um acht deutsche Infrastrukturprojekte zwischen 1970 und 2000}

Die Studie orientiert sich an den „Standards guter Praxis“ für QCA (Schneider und Wagemann 2010). Daher beginnt die Analyse mit einer Untersuchung der notwendigen Bedingungen. Erst anschließend folgt die Untersuchung der hinreichenden Bedingungen (Schneider und Wagemann 2012). Bei der Datentabelle fällt auf (Tab. 2), dass bis auf die Bedingung ideologisierte Gruppen alle Bedingungen eine schiefe Verteilung aufweisen. Dies kann beim Test auf notwendige Bedingungen dazu führen, dass Bedingungen als notwendig klassifiziert werden, obwohl sie trivial sind (Skewedness; Schneider und Wagemann 2012, S. 232-237). Es ist allerdings festzuhalten, dass die Bedingungen Baustart, Polizeigewalt und grundlegender Technikkonflikt und ebenso der Outcome in der Empirie prinzipiell eine schiefe Verteilung aufweisen. Der Baustart ist nur ein kurzer Moment in einem Prozess der Realisierung eines Infrastrukturprojektes und auch Polizeigewalt kommt in Verfassungsstaaten selten vor. Dies gilt ebenso für Protestgewalt. Und auch die meisten Infrastrukturkonflikte sind nicht von einer grundsätzlichen Auseinandersetzung über die verwendete Technologie geprägt. Sollten sich diese Bedingungen und der Outcome einer Normalverteilung annähern, wäre zu hinterfragen, ob die Fälle noch in ein demokratisches politisches System eingebettet sind und ob dann nicht ganz andere kausale Mechanismen und Bedingungen gewaltsamen Protest verursachen.

\subsection{Notwendige Bedingungen für Protestgewalt}

Für den Test auf notwendige Bedingungen wird allgemein ein höherer Konsistenzwert verlangt als für den Test auf hinreichende Bedingungen. Aus diesem Grund liegt der von uns gesetzte Schwellenwert bei 0,9 (Ragin 2006). Der Grad der Ab- 
Tab. 3 Test auf notwendige Bedingungen für gewaltsamen Protest. (Quelle: Eigene Daten, fsQCA 3.0)

\begin{tabular}{llllll}
\hline Bedingung & Konsistenz & $\begin{array}{l}\text { Ab- } \\
\text { deckung }\end{array}$ & Komplement & Konsistenz & $\begin{array}{l}\text { Ab- } \\
\text { deckung }\end{array}$ \\
\hline STARTCONS & 0,67 & 0,78 & $\sim$ STARTCONS & 0,33 & 0,11 \\
POLVIO & 0,37 & 0,87 & $\sim$ POLVIO & 0,70 & 0,20 \\
IDEOLOG & 0,71 & 0,45 & $\sim$ IDEOLOG & 0,37 & 0,16 \\
TECHCON & 0,44 & 0,40 & $\sim$ TECHCON & 0,56 & 0,20 \\
STARTCONS+POLVIO & 0,81 & 0,77 & $\sim$ STARTCONS + POLVIO & 0,33 & 0,11 \\
IDEOLOG+ TECHCON & 0,81 & 0,40 & $\sim$ IDEOLOG+ TECHCON & 0,66 & 0,21 \\
\hline
\end{tabular}

STARTCONS $=$ Baustart, $P O L V I O=$ Polizeigewalt, IDEOLOG $=$ ideologisierte Gruppen, TECH CON $=$ grundlegender Technikkonflikt,$+=$ logisches ODER

deckung ist nur für tatsächlich notwendige Bedingungen relevant. Demnach sind keine der untersuchten Bedingungen oder ihr Komplement notwendig für gewaltsamen Protest bei Konflikten um große Infrastrukturprojekte (Tab. 3). Alternativ können auch Kombinationen von Bedingungen mithilfe des logischen ODER verknüpft und getestet werden. Dies ist sinnvoll bei funktionalen Äquivalenten, deren einzelne Bedingungen nur in ihrer Gesamtheit notwendig für den Outcome sind. In unserem Modell sind der Baustart und Polizeigewalt sowie ideologisierte Gruppen und ein grundlegender Technikkonflikt jeweils funktionale Äquivalente. Sie können mithilfe der ODER-Funktion in fsQCA $3.0(+)$ ebenfalls getestet werden. Auch diese Ergebnisse scheitern am Schwellenwert. Sie sind jeweils mit einer Konsistenz von 0,81 allerdings deutlich höher als alle anderen Bedingungen.

Die Logik notwendiger Bedingungen besteht darin, dass bei Auftreten des Outcomes diese immer vorliegen. Gleichzeitig können notwendige Bedingungen im Gegensatz zu hinreichenden Bedingungen auch vorliegen, wenn der Outcome nicht realisiert ist. Unter dieser Maßgabe fällt bei der Betrachtung der Datentabelle auf, dass sich der Fall München I von den anderen Fällen abhebt. Er führt sowohl in seiner Konfiguration selbst als auch beim Test auf Notwendigkeit der funktionalen Äquivalente zu einem logischen Widerspruch.

München I zeigt, dass es noch vor Baustart und ohne Einsatz von Gewalt durch die Polizei zu Protestgewalt kam. Auch handelte es sich beim Infrastrukturprojekt Großflughafen München nicht um einen Konflikt, der in eine grundlegende Auseinandersetzung um die verwendete Technologie eingebettet war. Bei einer Reanalyse dieser Untersuchungseinheit kann festgestellt werden, dass die gewaltsame Eskalation mit der speziellen Situation vor Ort zusammenhing. 1974 kam es zu einem kollektiven Sit-in in einem Verwaltungsbüro, bei dem Bürgerinnen und Bürger gemeinschaftlich im Rahmen des Planfeststellungsverfahrens ausgelegte Akten zerstörten und entwendeten. Dieser und andere Fälle von Gewalt fanden noch vor Baustart in einer stark emotionalisierten Situation statt (Kretschmer 1984). Gewalteskalation kann im Rahmen des Modells auch durch weitere, bisher nicht erkannte Ereignisse ausgelöst werden. Ebenso können auch bisher unerkannte Bedingungen als gewaltlegitimierende Ressourcen zur Wirkung kommen. Da aufgrund mangelnder Informationen nicht mehr nachvollzogen werden kann, welcher konkrete Auslöser diese Emotionalisierung bewirkte, ist eine Erweiterung des analytischen Rahmens nicht möglich. 
Bei theoriebasiertem Ausschluss von München I beim Test auf Notwendigkeit ergeben sich für die funktionalen Äquivalente Konsistenzwerte in Höhe von jeweils 0,90. ${ }^{1}$ Sie erreichen damit knapp den Schwellenwert. Ereignisse und Werte des Modells der gewaltsamen Eskalation von Protest sind damit notwendige Bedingungen. Das Abdeckungsmaß in Höhe von 0,77 für Ereignisse und 0,40 für Werte zeigt, dass den Ereignissen im Prozess der gewaltsamen Eskalation von Protest ein höherer Stellenwert zukommt. Aufgrund des Fallausschlusses und der Konsistenzwerte sollte jedoch mit den Ergebnissen vorsichtig umgegangen werden.

\subsection{Hinreichende Bedingungen der gewaltsamen Eskalation von Protest}

Die Logik hinreichender Bedingungen besagt, anders als bei Notwendigkeit, dass bei Vorliegen immer der Outcome realisiert sein muss. Es kann damit Fälle geben, bei denen der Outcome existiert, aber die Bedingung nicht. Ist eine Bedingung, aber nicht der Outcome in einem Fall vorhanden, ist sie damit nicht hinreichend. Bevor die Fälle dem Test unterzogen werden können, müssen ihre Fuzzy-Werte Idealtypen zugeordnet werden. Idealtypen sind dichotom. 1 zeigt die Zugehörigkeit eines Falles zu einem Idealtyp an. 0 bedeutet das Gegenteil. Der Fuzzy-Wert 0,5 fungiert dabei als Schwellenwert für die Zuordnung zu den Idealtypen. Die vier hier untersuchten Bedingungen ergeben - bei $2^{\mathrm{k}}$ - insgesamt 16 mögliche Konfigurationen.

Die Wahrheitstafel (Tab. 4) zeigt, dass 12 Konfigurationen empirisch vorliegen. Der Outcome für jede Konfiguration muss per Hand selbständig eingetragen werden. Dabei liefert die Konsistenz einer jeden Konfiguration einen Anhaltspunkt dafür, ob der Outcome realisiert ist. Schneider und Wagemann geben als Schwellenwert für diese Entscheidung einen Konsistenzwert in Höhe von 0,75 an. Unabhängig vom Konsistenzwert sollte vom Vorliegen des Outcomes Abstand genommen werden, wenn Widersprüche (true logical contradictory) in den Zeilen existieren (2012, S. 127). Widersprüche kommen in den Konfigurationen, bei denen der Outcome 1 ist, nicht vor. Der Outcome der logischen Rudimente in den Zeilen 13 bis 16 kann ebenfalls theoriebasiert vorgegeben werden. Es wird allerdings dem Algorithmus überlassen, nicht aufgetretene Fälle für die Minimalisierung des Lösungsterms zu verwenden, da der Outcome nicht eindeutig vorhersagbar ist. Die Verwendung von logischen Rudimenten setzt voraus, dass sie prinzipiell die Möglichkeit besitzen, in der Empirie realisiert zu werden. Dies ist hier der Fall. Der Cutoff-Wert liegt aufgrund der Verteilung der Fälle bei 1 .

Die Konfigurationen erzeugen bei der Minimierung die zwei möglichen Hauptimplikanten Polizeigewalt und ideologisierte Gruppen sowie Polizeigewalt und grundlegender Technikkonflikt für Zeile 11 (Online-Anhang C). Diese werden durch den Fall Wyhl IV repräsentiert. Da am Rande einer Großdemonstration mit 20.000 Teilnehmerinnen und Teilnehmern verschiedene Gruppen versuchten, den Bauplatz erneut zu besetzen, drängte die Polizei die Demonstrierenden unter Einsatz von Schlagstöcken zurück. Daraufhin kam es zu Flaschen- und Steinwürfen auf die Ein-

\footnotetext{
1 Die anderen Bedingungen und ihre Komplemente erreichen alle den Schwellenwert nicht. Eine Übersicht aller Konsistenzwerte und Abdeckungsmaße bei Ausschluss von München I findet sich im Online-Anhang B.
} 
Tab. 4 Wahrheitstafel (Quelle: Eigene Daten, fsQCA 3.0)

\begin{tabular}{llllllll}
\hline $\mathrm{Nr}$ & STARTCONS & POLVIO & IDEOLOG & TECHCON & Anzahl & Outcome & Konsistenz \\
\hline 1 & 0 & 0 & 0 & 0 & 10 & 0 & 0,14 \\
2 & 0 & 0 & 1 & 0 & 5 & 0 & 0,14 \\
3 & 0 & 0 & 0 & 1 & 2 & 0 & 0 \\
4 & 0 & 0 & 1 & 1 & 2 & 0 & 0,09 \\
5 & 1 & 0 & 0 & 0 & 1 & 0 & 0,23 \\
6 & 0 & 1 & 0 & 0 & 1 & 0 & 0,50 \\
7 & 1 & 0 & 1 & 0 & 1 & 1 & 1 \\
8 & 1 & 1 & 1 & 0 & 1 & 1 & 1 \\
9 & 1 & 0 & 0 & 1 & 1 & 1 & 0,77 \\
10 & 1 & 0 & 1 & 1 & 1 & 1 & 1 \\
11 & 0 & 1 & 1 & 1 & 1 & 1 & 0,88 \\
12 & 1 & 1 & 1 & 1 & 1 & 1 & 1 \\
13 & 1 & 1 & 0 & 0 & 0 & $?$ & - \\
14 & 1 & 1 & 0 & 1 & 0 & $?$ & - \\
15 & 0 & 1 & 1 & 0 & 0 & $?$ & - \\
16 & 0 & 1 & 0 & 1 & 0 & $?$ & - \\
\hline
\end{tabular}

STARTCONS = Baustart, POLVIO = Polizeigewalt, IDEOLOG = ideologisierte Gruppen, TECH$C O N=$ grundlegender Technikkonflikt, Outcome $=$ kollektive Protestgewalt, ? / - logical Remainder, Konsistenz $=$ raw consistency

satzkräfte. Unter den Gewalttätern und im Protest insgesamt fanden sich Mitglieder von K-Gruppen und radikalen Umweltgruppen (Sternstein 2013). Aus diesem Grund erfolgt die weitere Berechnung mit dem Hauptimplikanten Polizeigewalt und ideologisierte Gruppen.

Eine erste Analyse der Fälle unter Ausschluss von logischen Rudimenten ergibt drei komplexe Implikanten (Online-Anhang D). Dabei umfasst der für die gewaltsame Eskalation bei politischen Konflikten um große Infrastrukturvorhaben hinreichende Term Baustart, keine Polizeigewalt und grundlegender Technikkonflikt den Fall Wyhl III. Dieser schließt in der Zeit vom 17. Februar bis 19. Februar 1975 den Baustart und die erste Platzbesetzung zur Verhinderung des Weiterbaus ein. Eine genaue Betrachtung des empirischen Falles und der konkreten historischen Situation und Abläufe bis zur erfolgreichen ersten Platzbesetzung geben Anlass, logische Rudimente in die Analyse aufzunehmen. Entgegen der Aussage des Terms kommt der Polizeigewalt keine Relevanz zu. Die Zerstörung des Zaunes, welcher den Bauplatz vor den Demonstrantinnen und Demonstranten schützen sollte, war für diese das Mittel, um ihre Protestaktion erfolgreich durchzuführen. Polizeigewalt hätte nicht die Absicht zur Sachbeschädigung, sondern lediglich die erfolgreiche Tatausführung verhindern können und wäre damit nicht ursächlich für die registrierte Protestgewalt (Sternstein 2013, S. 37).

Tab. 5 weist auf Basis der logischen Rudimente drei ursächliche Kombinationen aus. Diese umfassen das Auftreten von Polizeigewalt und ideologisierten Gruppen, die Beteiligung dieser Gruppen am Protest zum Zeitpunkt des Baustarts und das symbolisch wirkmächtige Ereignis des Baustarts innerhalb eines grundlegenden Technikkonfliktes. Sowohl die Gesamtabdeckung in Höhe von 0,77 und die Gesamt- 
Tab. 5 Hinreichende Bedingungen für Protestgewalt - mit logischen Rudimenten. (Quelle: Eigene Daten, fsQCA 3.0; Parsomonious Solution)

\begin{tabular}{lllll}
\hline Hauptimplikant & Fälle & $\begin{array}{l}\text { Rohab- } \\
\text { deckung }\end{array}$ & $\begin{array}{l}\text { Alleinige } \\
\text { Abdeckung }\end{array}$ & $\begin{array}{l}\text { Konsis- } \\
\text { tenz }\end{array}$ \\
\hline $\begin{array}{l}\text { STARTCONS * IDEO- } \\
\text { LOG }\end{array}$ & $\begin{array}{l}\text { A20 III }(1,1) \\
\text { Gorleben III }(1,1) \\
\text { Startbahn West IV }(1,1) \\
\text { München IV }(0,7,1)\end{array}$ & 0,53 & 0,17 & 1 \\
STARTCONS * TECH- & $\begin{array}{l}\text { [Gorleben III }(1,1)] \\
\text { Wyhl III }(1,0,7)\end{array}$ & 0,34 & 0,10 & 0,89 \\
CON & $\begin{array}{l}\text { [München IV }(0,7,1)] \\
{[\text { Gorleben III }(0,8,1)]}\end{array}$ & 0,37 & 0,14 & 0,96 \\
POLVIO * IDEOLOG & $\begin{array}{l}\text { [Startbahn West IV }(0,8,1)] \\
\text { Wyhl IV }(0,8,0,7)\end{array}$ & & & \\
Gesamtabdeckung & & 0,77 & & \\
Gesamtkonsistenz & & 0,93 & & \\
\hline
\end{tabular}

Die mit [...] umrahmten Fälle werden durch einen anderen Hauptimplikanten adäquater repräsentiert $P O L V I O=$ Polizeigewalt, IDEOLOG $=$ ideologisierte Gruppen, STARTCONS = Baustart, TECHCON = grundlegender Technologiekonflikt

Tab. 6 Hinreichende Bedingungen für friedlichen Protest ( Outcome). (Quelle: Eigene Daten, fsQCA 3.0; Parsomonious Solution)

\begin{tabular}{|c|c|c|c|c|}
\hline Hauptimplikant & Fälle & $\begin{array}{l}\text { Rohab- } \\
\text { deckung }\end{array}$ & $\begin{array}{l}\text { Alleinige } \\
\text { Abdeckung }\end{array}$ & $\begin{array}{l}\text { Konsis- } \\
\text { tenz }\end{array}$ \\
\hline $\begin{array}{l}\sim \mathrm{IDEOLOG} * \sim \mathrm{TECH}- \\
\text { CON }\end{array}$ & $\begin{array}{l}\text { A20 I }(0,7,1) \text {, } \\
\text { A20 II }(0,7,0,7), \\
\text { A20 IV }(1,1) \\
\text { A24 I, II, III, IV }(1,1) \text {, } \\
\text { Stuttgart I }(1,0,7), \\
\text { München } 1(1,0,3), \\
\text { Startbahn West I, II }(1,1) \\
\text { TransrapidHH }(1,1)\end{array}$ & 0,58 & 0,07 & 0,90 \\
\hline $\begin{array}{l}\sim \text { STARTCONS * } \sim \text { POL- } \\
\text { VIO }\end{array}$ & $\begin{array}{l}\text { A20 I, IV }(1,1) \text {, } \\
\text { A24 I, II, IV }(1,1) \text {, } \\
\text { Stuttgart I, II }(1,0.7) \text {, } \\
\text { Gorleben I, II }(1,1) \text {, } \\
\text { München I }(1,0.3) \text {, } \\
\text { München II, III, V }(1,1) \\
\text { Startbahn West I, II, III }(1,1) \text {, } \\
\text { TransrapidHH }(1,1) \text {, } \\
\text { Wyhl I, II }(1,1)\end{array}$ & 0,92 & 0,40 & 0,93 \\
\hline Gesamtabdeckung & & 0,98 & & \\
\hline Gesamtkonsistenz & & 0,92 & & \\
\hline
\end{tabular}

$\sim P O L V I O=$ keine Polizeigewalt, $\sim$ IDEOLOG $=$ keine ideologisierten Gruppen, $\sim$ STARTCONS $=$ kein Baustart, $\sim T E C H C O N=$ kein grundlegender Technologiekonflikt

konsistenz in Höhe von 0,93 sind (sehr) hoch. Der Baustart unter Beteiligung von ideologisierten Gruppen deckt dabei die meisten Fälle ab. Die alleinige Abdeckung von allen Konfigurationen ist sehr gering. Deutlich wird, dass dem dramatischen Ereignis des Baustarts und den ideologisierten Gruppen eine besondere Bedeutung für die gewaltvolle Eskalation von Konflikten um große Infrastrukturprojekte zukommt. 
Tab. 6 zeigt den Lösungsterm für das nicht Auftreten von Gewalt. Proteste um Infrastrukturprojekte eskalieren in zwei Fällen nicht. Zum einen gibt es keine gewaltsame Eskalation des Protestes, wenn der Konflikt nicht in einen grundlegenden Technikkonflikt eingebettet ist und keine ideologisierte Gruppen daran teilnehmen. Zum anderen gibt es keine Protestgewalt, wenn es bei der Realisierung des Bauprojektes keinen Baustart und keine Polizeigewalt gibt. Sowohl Rohabdeckung als auch alleinige Abdeckung des zweiten Terms ist deutlich größer.

\subsection{Ergebnisse}

Baustart, Polizeigewalt, ideologisierte Gruppen und ein grundlegender Technikkonflikt können die gewaltsame Eskalation von Protest in Konflikten um große Infrastrukturprojekte in Deutschland zwischen 1970 und 2000 erklären. Dabei beschreiben diese Bedingungen drei Pfade, auf denen Gewalt äquifinal verursacht wird. Hypothese 1, ein Baustart unter Beteiligung von ideologisierten Gruppen am Konflikt, ist dabei der bedeutsamste Pfad für die Konflikteskalation (Gesamtabdeckung 0,58). Der Baustart als wirkmächtiges Symbol für das Scheitern des Protestes im Verlauf von Bauprozessen ist ein Grund dafür. Beteiligen sich dann Gruppen am Protest, die den Entscheidungsprozessen im politischen System ihre Legitimität absprechen, erscheint Gewalt als letztes legitimes Mittel zur Durchsetzung der Ziele des Protestes.

Sowohl im Konflikt um die Ostseeautobahn A20 als auch um den Großflughafen München fungierte der Baustart als symbolisch wirkmächtiges Ereignis, das für die Realisierung des Projektes steht und verstärkt negative Affekte auslöste. In der politischen Auseinandersetzung sind gewaltfreie Mittel konventioneller Partizipation wie das Sammeln von Unterschriften oder die Einreichung von Klagen vor den Verwaltungsgerichten ausgeschöpft. Das Bauprojekt kann auf diese Weise nicht mehr verhindert werden. Die im Geheimen zweckrational eingesetzte Sachbeschädigung lässt sich dann ideologisch legitimieren. Zum anderen finden sich mit Gorleben und Startbahn West Fälle, in denen zum Baustart Massen mobilisiert werden konnten und Gewalt in Form von Sachbeschädigungen zur Verhinderung des Bauprojektes und zur Symbolisierung des Protestes von ideologisierten Gruppen in Konflikten eingesetzt wurden. Durch den vorhandenen Resonanzraum können allerdings die Sachbeschädigungen auch in aller Öffentlichkeit durchgeführt werden.

Neben diesem Pfad besitzen die Pfade Baustart in einem grundlegenden Technikkonflikt und die Eskalation durch Polizeigewalt bei Konflikten unter Beteiligung ideologisierter Gruppen Relevanz. Damit finden Hypothese 2 und 3 ihre Bestätigung in der vorliegenden Untersuchung. Hypothese 4 konnte aufgrund begrenzter empirischer Vielfalt nicht bestätigt werden. Die Ergebnisse geben allerdings Anlass, an dieser Hypothese festzuhalten und in weiteren Untersuchungen zu überprüfen. Hierfür spricht auch die Untersuchung der Bedingungen für friedlichen Protest in Konflikten um Infrastrukturprojekte. Zwei Pfade führen zu dieser Wirkung. Zum einen verursacht das Fehlen von Baustart und Polizeigewalt friedlichen Protest. Zum anderen bleiben Proteste friedlich, wenn der Konflikt nicht in einen grundlegenden Technikkonflikt eingebettet ist und sich keine ideologisierten Gruppen dem Protest 
anschließen. Hypothese 4 kann keinem dieser zwei Pfade zugeordnet werden und sollte somit Gewalt nach sich ziehen.

Gleichzeitig bilden beide Pfade jeweils eigene, von uns bereits oben kurz angerissene Gruppen von Bedingungen, welche als funktionale Äquivalente fungieren. Ideologisierte Gruppen und ein grundlegender Technikkonflikt können dabei als Eskalationspotenzial interpretiert werden. Wenn mindestens eine von beiden Bedingungen vorliegt, dann muss der Konflikt nicht zwingend in Gewalt eskalieren. Es reicht allerdings ein Funken aus, der einen Flächenbrand im Konflikt auslösen kann. Als solch einen Eskalationsfunken können die Ereignisse Baustart und Polizeigewalt interpretiert werden. Jeweils einzeln setzen sie bei vorhandenem Potenzial einen Mechanismus in Gang, an dessen Ende der Protest seinen friedlichen Charakter verliert. Gleichzeitig zeigen die geringen Werte der alleinigen Abdeckung genau diese Verschränkung der beiden Gruppen von Bedingungen. Jeweils eine Bedingung aus jeder Gruppe bildet einen Pfad, der zur gewaltsamen Eskalation von Protest führt. Insofern wäre Hypothese 4 folgerichtig und in zukünftigen Studien zu überprüfen.

\section{Fazit}

Politische Konflikte um große Infrastrukturprojekte führten in der Bundesrepublik Deutschland wiederholt zu Gewalteskalation auf Seiten der Protestierenden. Dabei erklären drei verschiedene Pfade diese Eskalation. Gewalt verursachend wirken sich der Baustart in Verbindung mit einem grundlegenden Technikkonflikt oder unter Beteiligung ideologisierter Gruppen aus. Daneben führen die Interaktionen zwischen Polizei und ideologisierten Gruppen ebenfalls zu Protestgewalt. In der Untersuchung bestätigte sich, dass die gewaltsame Eskalation von den Rahmenbedingungen des Konfliktes abhängt. Im Laufe eines solchen Prozesses wirken Ereignisse in Form des Baustarts oder der Polizeigewalt wie ein Eskalationsfunken, der Eskalationspotenziale in Form von ideologisierten Gruppen oder grundlegendem Technikkonflikt entfacht und zur gewaltsamen Eskalation dieser Konflikte beiträgt.

Die Ergebnisse unserer Untersuchung sind konsistent und erzielen eine hohe Abdeckung, allerdings können sie Fälle wie München I nicht erklären. Dieser ist davon geprägt, dass weder ein bisher erkanntes Eskalationspotenzial noch ein Eskalationsfunken vorlagen. Diese Konfiguration bildet damit einen Spezialfall, welcher zukünftig näher zu untersuchen ist. Auch weitere Bedingungen sowohl in Form von Eskalationspotenzialen als auch in Form von Eskalationsfunken sind möglich. Weitere Forschung kann dazu dienen, diese zu identifizieren. Ebenfalls wäre zu zeigen, inwiefern unser Modell auf andere politische Konflikte übertragen werden kann. Beispiele wie die Untersuchung rechtsextremer politischer Gewalt auf Basis der Deprivationstheorie (Rippl und Baier 2005) zeigen, dass der von uns angenommene kausale Mechanismus gewaltsamer Eskalationen auch in anderen konfliktbehafteten Politikbereichen wirksam ist. Insofern sollten zur Erklärung von Protestgewalt auch in diesen Fällen Eskalationspotenziale und Eskalationsfunken auf der Mesoebene identifizierbar sein.

Wie können gewaltsame Eskalationen von politischen Konflikten um große Infrastrukturprojekte in Deutschland verhindert werden? Aus den Ergebnissen las- 
sen sich zwei Empfehlungen ableiten: Zum einen zeigen Attribuierungsprozesse, dass mit der Implementierung von Verfahren der frühzeitigen Öffentlichkeitsbeteiligung in das Verwaltungsverfahrensgesetz 2013 und einer faktischen Übererfüllung von Partizipationserfordernissen des EU-Rechts in Deutschland (Fink und Ruffing 2017) ein erster Schritt zur Minimierung von Eskalationspotenzialen getan wurde. Zukünftig wird es - insbesondere bei grundlegenden Technikkonflikten - wichtig sein, alle relevanten Gruppen in den Prozess einzubeziehen, um einer gegenseitigen negativen Attribuierung der Konfliktgegner entgegenzuwirken und so das Eskalationspotenzial zu minimieren (Fraune und Knodt 2017). Die aktuellen Ereignisse um den Hambacher Forst sind ein Beleg dafür und zeigen die Relevanz weiterer Forschung. Zum anderen müssen in stark eskalierten Konflikten konsequent polizeiliche Deeskalationsstrategien angewandt und Fälle polizeilichen Fehlverhaltens zwingend geahndet werden, um der (gruppenbezogenen) Legitimierung von Protestgewalt auf der Grundlage negativer Attribuierungen entgegenzuwirken. Zusammenfassend gilt es zu vermeiden, dass sich Eskalationspotenziale durch einen Funken entzünden und zu einem Flächenbrand ausweiten, der sich nur noch schwer und mit erheblichem Ressourcenaufwand löschen lässt.

Danksagung Ralf Keil, Derk Trei und André Völker haben die Recherche für diese Studie sehr engagiert unterstützt. Dafür danken wir ihnen ausdrücklich.

Open Access Dieser Artikel wird unter der Creative Commons Namensnennung 4.0 International Lizenz (http://creativecommons.org/licenses/by/4.0/deed.de) veröffentlicht, welche die Nutzung, Vervielfältigung, Bearbeitung, Verbreitung und Wiedergabe in jeglichem Medium und Format erlaubt, sofern Sie den/die ursprünglichen Autor(en) und die Quelle ordnungsgemäß nennen, einen Link zur Creative Commons Lizenz beifügen und angeben, ob Änderungen vorgenommen wurden.

Förderung Diese Studie wurde mit Mitteln des „Niedersächsischen Vorab“ der VolkswagenStiftung gefördert.

\section{Literatur}

Arendt, Hannah. 1995. Macht und Gewalt. München: Piper.

Bandelow, Nils C., und Barbara Thies. 2014. Gerechtigkeitsempfindungen bei Großprojekten als Ursache von Konflikteskalationen? Vertrauen und Legitimität als moderierende Faktoren illustriert am Beispiel der Konflikte um die Erweiterung des Frankfurter Flughafens. Politische Psychologie/Journal of Political Psychology 3:24-37.

Beier, Harald. 2016. Wie wirken „Subkulturen der Gewalt“? Das Zusammenspiel von Internalisierung und Verbreitung gewaltlegitimierender Normen in der Erklärung von Jugendgewalt. KZfSS Kölner Zeitschrift für Soziologie und Sozialpsychologie 68:457-485.

Berkowitz, Leonard. 1993. Aggression. Its Causes, Consequences and Control. New York: McGraw-Hill.

Bosi, Lorenzo. 2006. The Dynamics of Social Movement Development: Northern Ireland's Civil Rights Movement in The 1960s. Mobilization 11:81-100.

Brettschneider, Frank, und Wolfgang Schuster (Hrsg.). 2013. Stuttgart 21. Ein Großprojekt zwischen Protest und Akzeptanz. Wiesbaden: Springer VS.

Caren, Neal, und Aaron Panofsky. 2005. TQCA. A Technique for Adding Temporality to Qualitative Comparative Analysis. Sociological methods \& research 34:147-172.

Coleman, James S. 1994. Foundations of social theory. Cambridge: Belknap Press of Harvard Univ. Press.

Della Porta, Donatella. 1995. Social Movements, Political Violence, and the State. A Comparative Analysis of Italy and Germany. Cambridge: Cambridge University Press.

Della Porta, Donatella. 2008. Research on Social Movements and Political Violence. Qualitative sociology 31:221-230.

Della Porta, Donatella. 2013. Clandestine Political Violence. Cambridge: Cambridge University Press. 
Della Porta, Donatella, und Herbert Reiter (Hrsg.). 1998. Policing Protest. The Control of Mass Demonstrations in Western Democracies. Minneapolis, London: University of Minnesota Press.

Dul, Jan, Tony Hak, Gary Goertz, und Chris Voss. 2010. Necessary Condition Hypotheses in Operations Management. International journal of operations \& production management 30:1170-1191.

Enzmann, Birgit. 2013. Politische Gewalt. Formen, Hintergründe, Überwindbarkeit. In Handbuch Politische Gewalt. Formen - Ursachen - Legitimation - Begrenzung, Hrsg. Birgit Enzmann, 43-66. Wiesbaden: Springer VS.

Fink, Simon, und Eva Ruffing. 2017. The Differentiated Implementation of European Participation Rules in Energy Infrastructure Planning. Why Does the German Participation Regime Exceed European Requirements? European Policy Analysis 3:274-294. https://doi.org/10.1002/epa2.1026.

Fraune, Cornelia, und Michèle Knodt. 2017. Challenges of Citizen Participation in Infrastructure PolicyMaking in Multi-Level Systems-The Case of Onshore Wind Energy Expansion in Germany. European Policy Analysis 3:256-273. https://doi.org/10.1002/epa2.1022.

Glasl, Friedrich. 2013. Konfliktmanagement. Ein Handbuch für Führungskräfte Beraterinnen und Berater. Bern, Stuttgart: Haupt; Verl. Freies Geistesleben.

Goodwin, Jeff, und James M. Jasper. 1999. Caught in a Winding, Snarling Vine: The Structural Bias of Political Process Theory. Sociological Forum 14:27-54.

Goodwin, Jeff, Francesca Polletta, und James M. Jasper. 2001. Passionate Politics. Emotions and Social Movements. Chicago: University of Chicago Press.

Graham, Hugh D., und Ted Robert Gurr. 1969. Violence in America: Historical and Comparative Perspectives. Bd. 2. Washington, DC: US Government Printing Office.

Hak, Tony, Jaspers, Ferdinand, und Jan Dul. 2013. The Analysis of Temporally Ordered Configurations: Challenges and Solutions. In Fiss, Peer C., Cambré, Bart und Marx, Axel. (Hrsg.), Configurational Theory and Methods in Organizational Research (Research in the Sociology of Organizations, Vol. 38), Emerald Group Publishing Limited, Bingley, Chapter 5, 107-127. https://doi.org/10.1108/ S0733-558X(2013)0000038009.

Hino, Airo. 2009. Time-Series QCA: Studying Temporal Change Through Boolean Analysis. Sociological Theory and Methods 24:247-265.

Hirschle, Jochen. 2015. Soziologische Methoden. Eine Einführung. Weinheim: Beltz Juventa.

Irvin, Cynthia L. 1999. Militant Nationalism. Between Movement and Party in Ireland and The Basque Country. Minneapolis: University of Minnesota Press.

Jasper, James M. 1998. The Emotions of Protest: Affective and Reactive Emotions in and Around Social Movements. Sociological Forum 13:397-424.

Jasper, James M. 2006. Motivation and Emotion. In The Oxford Handbook of Contextual Political Analysis, Hrsg. Robert E. Goodin, Charles Tilly, 157-171. Oxford: Oxford University Press.

Kaufer, Ricardo, und Paula Lein. 2018. Widerstand im Hambacher Forst: Analyse einer anarchistischen Waldbesetzung. http://forschungsjournal.de/sites/default/files/fjsbplus/fjsb-plus_2018-4_kaufer_ lein.pdf. Zugegriffen: 29.08.2019.

Koopmans, Ruud, Paul Stathan, Marco Giugni, und Florence Passy. 2008. Contested Citizenship. Immigration and Cultural Diversity in Europe. Minneapolis: Univ. of Minnesota Press.

Kretschmer, Winfried. 1984. Fallstudie: Großflughafen München II. In Flughafenprojekte als Politikum. Die Konflikte in Stuttgart, München und Frankfurt, Hrsg. Dieter Rucht, 100-194. Frankfurt am Main: Campus.

Kriesi, Hanspeter. 1989. The Political Opportunity Structure of the Dutch Peace Movement. West European Politics 12:295-312.

Lamnek, Siegfried. 2002. Individuelle Rechtfertigungsstrategien von Gewalt. In Internationales Handbuch der Gewaltforschung, Hrsg. Wilhelm Heitmeyer, John Hagan, 1379-1396. Wiesbaden: VS.

Mayntz, Renate. 2009. Sozialwissenschaftliches Erklären. Probleme der Theoriebildung und Methodologie. Frankfurt am Main: Campus.

McAdam, Doug. 1983. Tactical Innovation and the Pace of Insurgency. American Sociological Review 48:735.

Neidhardt, Friedhelm. 1986. Gewalt - Soziale Bedeutungen und sozialwissenschaftliche Bestimmungen des Begriffs. In Was ist Gewalt? Auseinandersetzungen mit einem Begriff, Sonderband, BKA-Forschungsreihe, Hrsg. Volker Krey, Friedhelm Neidhardt, 109-147. Wiesbaden: Bundeskriminalamt.

Neidhardt, Friedhelm, und Dieter Rucht. 2001. Protestgeschichte der Bundesrepublik Deutschland 1950-1994. Ereignisse, Themen, Akteure. In Protest in der Bundesrepublik. Strukturen und Entwicklungen, Hrsg. Dieter Rucht, 27-70. Frankfurt am Main, New York: Campus.

Ragin, Charles C. 1987. The Comparative Method. Moving Beyond Qualitative and Quantative Strategies. Berkeley: Univ. of California Press. 
Ragin, Charles C. 2000. Fuzzy-Set Social Science. Chicago: University of Chicago Press.

Ragin, Charles C. 2006. Set Relations in Social Research: Evaluating Their Consistency and Coverage. Political Analysis 14:291-310.

Ragin, Charles C., und Sarah Ilene Strand. 2008. Using Qualitative Comparative Analysis to Study Causal Order: Comment on Caren and Panofsky (2005). Sociological Methods and Research 36:431-441.

Rippl, Susanne, und Dirk Baier. 2005. Das Deprivationskonzept in der Rechtsextremismusforschung. KZfSS Kölner Zeitschrift für Soziologie und Sozialpsychologie 57:644-666.

Rucht, Dieter. 2002. Gewalt und neue soziale Bewegungen. In Internationales Handbuch der Gewaltforschung, Hrsg. Wilhelm Heitmeyer, John Hagan, 461-478. Wiesbaden: VS.

Rucht, Dieter, und Simon Teune. 2017. Einleitung: Das Protestgeschehen in der Bundesrepublik seit den 1980er Jahren zwischen Kontinuität und Wandel. In Protest in Bewegung? Zum Wandel von Bedingungen Formen und Effekten politischen Protests, Hrsg. Priska Daphi, 9-33. Baden-Baden: Nomos.

Rucht, Dieter, und Simon Teune. o. J. PRODAT - Dokumentation und Analyse von Protesereignissen in der Bundesrepublik. https://www.wzb.eu/system/files/docs/sine/prodat_1950-2002.sav. Zugegriffen: 23. September 2018.

Schmid, Jeannette. 2002. Die Konflikttheorie der Aggressionstheorie. In Sozialwissenschaftliche Konflikttheorien. Eine Einführung, Hrsg. Thorsten Bonacker, 507-526. Opladen: Leske + Budrich.

Schneider, Carsten Q., und Claudius Wagemann. 2010. Standards of Good Practice in Qualitative Comparative Analysis (QCA) and Fuzzy-Sets. Comparative sociology 9:397-419.

Schneider, Carsten Q., und Claudius Wagemann. 2012. Set-Theoretic Methods For the Social Sciences. A Guide to Qualitative Comparative Analysis. Cambridge: Cambridge University Press.

Snow, David A., und Pamela E. Oliver. 1995. Social Movements and Collective Behavior: Social Psychological Dimensions and Considerations. In Sociological Perspectives on Social Psychology, Hrsg. Karen S. Cook, 571-599. Boston: Allyn and Bacon.

Snow, David A., Burke E. Rochford Jr., Steven K. Worden, und Robert D. Benford. 1986. Frame Alignment Processes, Micromobilization, and Movement Participation. American Sociological Review 51:464-481.

Sternstein, Wolfgang. 2013. „Atomkraft-nein danke!“. Der lange Weg zum Ausstieg. Frankfurt am Main: Brandes \& Apsel.

Taylor, Verta, und Nancy E. Whittier. 1992. Collective Identity in Social Movement Communities: Lesbian Feminist Mobilization. In Frontiers in Social Movement Theory, Hrsg. Aldon D. Morris, Carol M. Mueller, 104-129. New Haven: Yale University Press.

Thaa, Winfried. 2013. „Stuttgart 21“ - Krise oder Repolitisierung der repräsentativen Demokratie? Politische Vierteljahresschrift 54:1-20.

Tilly, Charles. 1978. From Mobilization to Revolution. New York: McGraw-Hill.

Tilly, Charles. 2001. Mechanisms in Political Processes. Annual Review of Political Science 4:21-41.

Virgl, Christoph J. 2011. Protest in der Weltgesellschaft. Wiesbaden: VS.

Vogeler, Colette S., und Nils C. Bandelow. 2016. Devil Shift und Angel Shift in eskalierten politischen Konflikten. Zeitschrift für Politikwissenschaft 26:301-324.

Vogeler, Colette S., und Nils C. Bandelow. 2018. Mutual and Self Perceptions of Opposing Advocacy Coalitions. Devil Shift and Angel Shift in a German Policy Subsystem. Review of Policy Research. https://doi.org/10.1111/ropr.12299.

Waldmann, Peter (Hrsg.). 1993. Beruf: Terrorist. Lebensläufe im Untergrund. München: Beck.

White, Robert W. 1993. Provisional Irish Republicans. An Oral and Interpretive History. Westport: Greenwood.

Zimmermann, Ekkart. 1998. Ressourcenmobilisierung und Gewalt. Forschungsjorunal Neue Soziale Bewegungen 11:55-67.

Zwerman, Gilda, Patricia Steinhoff, und Donatella Della Porta. 2000. Disappearing Social Movements: Clandestinity in The Cycle of New Left Protest in The U.S., Japan, Germany, and Italy. Mobilization 5:85-104. 